\title{
Grenzen der Hilfeleistungspflicht des Notarztes im öffentlichen Rettungsdienst
}

\author{
Felix Geser
}

Korrespondenzadresse und Autorenhinweis:

Dr. med. univ. Felix Geser, PhD

Facharzt für Neuropathologie

Jahnstr. 70.

73037 Göppingen

E-mail: felix.geser@alumni.i-med.ac.at

Es bestehen keine Interessenkonflikte. 


\section{Einleitung}

Das Thema der folgenden Arbeit besteht in ausgewählten Grenzen der Hilfeleistungspflicht des Notarztes im öffentlichen Rettungsdienst in Baden-Württemberg. Der Hintergrund dieser Thematik ist die Situation, dass die Tätigkeiten im Notarzt- und Rettungsdienst häufig mit persönlichen Gefährdungen verbunden sind. Im Raum steht aber auch immer die Problematik von Gefährdungssituationen, die durch den Notarzt „nur“ als solche wahrgenommen, sozusagen ,empfunden“ werden. Es erhebt sich jedenfalls prinzipiell die Frage, ob und inwieweit ein Notarzt zum Handeln verpflichtet ist, wenn sich beispielsweise bei einer Amoklage die polizeiliche Situation noch unübersichtlich darstellt und der zuständige Einsatzführer der vor Ort tätigen Polizeikräfte das Rettungsteam wegen der unstreitig dringend notwendigen Versorgung von lebensbedrohlich Verletzten (Schussverletzung) unter Gewährung von Polizeischutz in das Objekt entsenden möchte.

Die Aufgabe des Rettungsdienstes ist die Notfallrettung bzw. der damit verbundene Transport der Kranken/Verletzen. Notfallpatienten sind gemäß $\S 1$ Rettungsdienstgesetz (RDG) von Baden-Württemberg, Abs. 2 „Kranke oder Verletzte, die sich in Lebensgefahr befinden oder bei denen schwere gesundheitliche Schäden zu befürchten sind, wenn sie nicht umgehend medizinische Hilfe erhalten."1 M. R. Ufer $(1999)^{2}$ unterschied neben der fehlenden Erforderlichkeit und Unzumutbarkeit der ärztlichen Hilfeleistung noch folgende Grenzen der Notfallmedizin: 1. Biologische oder räumliche Grenzen (Grenzen der Natur der Sache), 2. Schicksalhafte Verläufe, 3. Rechtfertigende/entschuldigende Pflichtenkollisionen und 4. Der einer Behandlung entgegenstehende Wille des Patienten (sofern beachtlich).

Gemäß RDG $\S 10$, Abs. 1 nehmen am Rettungsdienst geeignete Ärzte teil, wobei die Eignungsvoraussetzungen durch das Satzungsrecht der Landesärztekammern bestimmt werden. Die Weiterbildungsordnung der Landesärztekammer Baden-Württemberg (WBO) weist explizit darauf hin, dass mit der Zuerkennung der Zusatzbezeichnung Notfallmedizin „der Nachweis der Eignung im Sinne des $\S 10$ Abs. 1 Satz 1 des Rettungsdienstgesetzes geführt“3 ist. Gemäß WBO beinhaltet die Zusatzweiterbildung Notfallmedizin das Erkennen von drohenden oder eingetroffenen Notfällen und die Behandlung in Notsituationen sowie die Wie-

\footnotetext{
${ }^{1}$ Vgl. Gesetz über den Rettungsdienst in der Fassung vom 8. Februar 2010, GB1. 2010, S. 285ff.

${ }^{2}$ Vgl. UFER (1999), S. 4ff.

${ }^{3}$ WBO vom 15. März 2006, Sonderausgabe Ärzteblatt Baden-Württemberg 4, 2006, S. 103, geändert durch Satzung der Landesärztekammer Baden-Württemberg zur Änderung der Weiterbildungsordnung vom 20.12.2006 (ÄBW 2007, S. 64).
} 
derherstellung und Erhaltung akut gefährdeter Vitalparameter. Die Voraussetzungen zum Erwerb der Bezeichnung „Notfallmedizin“ umfassen 2 Jahre Weiterbildung in der direkten Versorgung von Patienten in einem Krankenhaus, bei dem 24 Stunden-Notfallaufnahmebereitschaft mit mannigfaltigen akuten stationären Fällen besteht; davon müssen 6 Monate intensivmedizinische Weiterbildung an einer zugelassenen Weiterbildungsstätte unter verantwortlicher Leitung der von der Bezirksärztekammer befugten Ärzte (gemäß WBO § 5, Abs. 1) absolviert werden. Es sind zudem 80 Unterrichteinheiten Kurs-Weiterbildung gemäß WBO § 4, Abs. 8 (d. h. Anerkennung eines Kurses und dessen Leiters durch die zuständige Bezirksärztekammer nach bundeseinheitlichen Empfehlungen) in allgemeiner und spezieller Behandlung von Notfallpatienten vorgesehen. Danach müssen 50 Notarzteinsätze unter Anleitung eines Arztes, berechtigt zum Führen der Zusatzbezeichnung „Notfallmedizin“, abgeleistet werden. Inhaltlich werden von der WBO Wissen, Erfahrung und Fähigkeiten in folgenden Gebieten gefordert: 1. Grundlegende rechtliche bzw. organisatorische Aspekte des Rettungsdienstes, 2. Diagnose und Therapie von akuten Beeinträchtigungen der Vitalfunktionen, 3. Notfallmedikation, 4. Fachgerechte Lagerung, 5. Erlangung der Transportfähigkeit und 6. Spezielle Aspekte beim Massenanfall von Notfallpatienten (inkl. Sichtung).

Gemäß RDG § 4, Abs. 1 wird ein Landesausschuss für den Rettungsdienst formiert, der aus einem Vertreter des Innenministeriums und je zehn Vertretern der Leistungsträger nach $\S 2$, Abs. 1 des RDG (i. e. Arbeiter-Samariter-Bund, dem Deutschen Roten Kreuz und seiner Bergwacht Württemberg, der Johanniter-Unfall Hilfe, dem Malteser-Hilfsdienst, DRF Luftrettung, der Bergwacht Schwarzwald, der Deutschen Lebensrettungsgesellschaft sowie bei Bedarf anderer Stellen) sowie dem Kostenträger besteht. Zudem gehören zu dem Landesausschuss andere Stellen mit beratender Stimme gemäß RDG § 4, Abs. 1, Satz $4^{4}$. Laut RDG $\S 3$, Abs. 1 fertigt das Innenministerium gemeinsam mit dem Landesausschuss für den Rettungsdienst einen Rettungsdienstplan an. Gemäß Rettungsdienstplan 2014 BadenWürttemberg (RDP) ist der Notarzt „ein im Rettungsdienst zur fachgerechten, notfallmedizinischen Versorgung von erkrankten und verletzten Personen tätiger Arzt, der über eine besondere Qualifikation verfügen muss. ${ }^{\text {(55 }}$

Dem Leitenden Notarzt obliegt gemäß RDG $§ 10$, Abs. 2 die Koordination der ärztlichen Versorgung bei Schadensereignissen mit einer Vielzahl von Verletzten oder Erkrankten. Gemäß

\footnotetext{
${ }^{4}$ Vgl. Gesetz über den Rettungsdienst in der Fassung vom 8. Februar 2010, GB1. 2010, S. 285ff., geändert durch Artikel 1 des Gesetztes vom 17. Dezember 2015 (GBl., S. 1182).

${ }^{5}$ RDP, S. 174 .
} 
RDP soll der Leitende Notarzt in der Funktion als Führungskraft des Rettungsdienstes bei einem Großschadenfall mit einem massenhaften Anfall von Betroffenen die medizinischen Maßnahmen entsprechend steuern, abstimmen und kontrollieren, damit möglichst alle Patienten medizinisch versorgt werden können; dies inkludiert u. a. die Festlegung des Brennpunktes und der Form des medizinischen Vorgehens durch Sichtung ${ }^{6}$. Ein Organisatorischer Leiter Rettungsdienst stellt eine Unterstützung für den Leitenden Notarzt bei Schadenslagen nach RDG $\S 10$, Abs. 2 (vgl. RDG $§ 10 a$ ) dar. Die niedergelassenen Ärzte nehmen ergänzend - d. h. bei Notwendigkeit - am Rettungsdienst teil (vgl. Kapitel VIII, Abschnitt 1 des RDP) ${ }^{7}$ Der Leitende Notarzt besitzt gemäß des RDP Weisungsbefugnis gegenüber den involvierten Ärzten für das medizinisch-einsatztaktische Vorgehen. Insofern nimmt er - beliehen durch die Bestellung durch den Oberbürgermeister beziehungsweise durch den Landrat des Stadt/Landkreises des entsprechenden Rettungsdienstbereiches - hoheitliche Tätigkeiten wahr. Diese Bestellung stellt die notwendige Vorbedingung für die Deckung des Haftungsrisikos des Leitenden Notarztes über die Amtshaftung des jeweiligen Landes dar ${ }^{8}$.

\section{Hauptteil}

\subsection{Sorgfaltspflicht des Arztes}

Die (Muster-)Berufsordnung für die in Deutschland tätigen Ärztinnen und Ärzte (MBO) ${ }^{9}$ sagt in $\S 1$, Abs. 1: „Der ärztliche Beruf ist kein Gewerbe. Er ist seiner Natur nach ein freier Beruf.“" ${ }^{\text {“10 }}$ Und in der Bundesärzteordnung (B ̈̈O) $§ 1$, Abs. 2 heißt es faktisch identisch: „Der ärztliche Beruf ist kein Gewerbe; er ist seiner Natur nach ein freier Beruf.“11 Das Partnerschaftsgesellschaftsgesetz $\S 1$, Abs. 2 äußert sich hinsichtlich der Freiberuflichkeit wie folgt:

\footnotetext{
${ }^{6}$ Vgl. RDP, S. 162.

${ }^{7}$ Vgl. RDP, S. 175 .

${ }^{8}$ Vgl. RDP, S. $162 \mathrm{f}$.

${ }^{9}$ Wie J. Heberer betonte, stellt die MBO (beschlossen durch den Deutschen Ärztetag) eine Orientierungslinie für die jeweiligen Berufsordnungen der Länder dar, um ein möglichst gleichförmiges Berufsrecht innerhalb der Bundesrepublik Deutschland zu gewährleisten; unmittelbare Rechtswirkung entfalten aber erst die Berufsordnungen der einzelnen Länder - vgl. HEBERER (2001), S. 226 und 358.

${ }^{10} \mathrm{MBO}$ in der Fassung des Beschlusses des 118. Deutschen Ärztetages 2015 in Frankfurt am Main, Deutsches Ärzteblatt 2015, 112:A 1348; DOI: 10.3238/arztebl.2015.mbo_daet2015, S. A 1-9, abrufbar unter: www.bundesaerztekammer.de/recht/berufsrecht/muster-berufsordnung-aerzte/muster-berufsordnung/ (zugegriffen am 23.05.2016).

${ }^{11} \mathrm{~B} \ddot{\mathrm{O} O}$ vom 16.04.1987 (BGB1. I, S. 1219).
} 
„Die freien Berufe haben im allgemeinen auf der Grundlage besonderer beruflicher Qualifikation oder schöpferischer Begabung die persönliche, eigenverantwortliche und fachlich unabhängige Erbringung von Dienstleistungen höherer Art im Interesse der Auftraggeber und der Allgemeinheit zum Inhalt. Ausübung eines Freien Berufs im Sinne dieses Gesetzes ist die selbständige Berufstätigkeit der Ärzte, Zahnärzte, Tierärzte, Heilpraktiker, Krankengymnasten, Hebammen, Heilmasseure, Diplom-Psychologen, Mitglieder der Rechtsanwaltskammern, Patentanwälte, Wirtschaftsprüfer, Steuerberater, beratenden Volks- und Betriebswirte, vereidigten Buchprüfer (vereidigte Buchrevisoren), Steuerbevollmächtigten, Ingenieure, Architekten, Handelschemiker, Lotsen, hauptberuflichen Sachverständigen, Journalisten, Bildberichterstatter, Dolmetscher, Übersetzer und ähnlicher Berufe sowie der Wissenschaftler, Künstler, Schriftsteller, Lehrer und Erzieher." ${ }^{\text {12 }}$

Die Übernahme der Behandlung verpflichtet den Arzt gemäß Sozialgesetzbuch V (SGB V) § 76, Abs. 4. dem Patienten gegenüber ,zur Sorgfalt nach den Vorschriften des bürgerlichen Vertragsrechts“"13. Entsprechendes findet sich im Bundesmantelvertrag-Ärzte (BMV-Ä), geschlossen zwischen der Kassenärztlichen Bundesvereinigung und dem Spitzenverband Bund der Krankenkassen: „Die Übernahme der Behandlung verpflichtet den Vertragsarzt dem Versicherten gegenüber zur Sorgfalt nach den Vorschriften des bürgerlichen Vertragsrechtes.“ “14 Und dies beinhaltet auch, dass der Vertragsarzt, der die Behandlung übernommen hat, verpflichtet ist, die in diesem Rahmen notwendigen Verordnungen von Leistungen zu treffen, soweit diese im Bereich der Leistungspflicht der gesetzlichen Krankenversicherung liegen (vgl. BMV-Ä $\S 13$, Abs. 8). Die MBO $\S 2$, Abs. 3 verpflichtet den Arzt zu einer gewissenhaften Ausübung seines Berufs und „erfordert insbesondere die notwendige fachliche Qualifikation und die Beachtung des anerkannten Standes der medizinischen Erkenntnisse.“ Gemäß Bürgerlichem Gesetzbuch (BGB) § 276, Abs. 2 bedeutet Fahrlässigkeit, „die im Verkehr erforderliche Sorgfalt “"15 nicht zu beachten. Behandlungsfehler sind, wie es die Beobachtung des ärztlichen Alltags zeigt, in der Regel nicht auf Vorsatz, d. h. auf wissentliches sowie willentliches Verursachen einer Schädigung zurückzuführen, sondern auf fahrlässiges Handeln i. S. einer Nichtbeachtung der anerkannten medizinisch-wissenschaftlichen Regeln ${ }^{16}$. Wissenschaftliche Fachgesellschaften (z. B. die Arbeitsgemeinschaft der Wissenschaftlichen

\footnotetext{
${ }^{12}$ Partnerschaftsgesellschaftsgesetz vom 25.07.1994 (BGB1. I, S. 1744f), § 1 geändert durch Artikel 1a des Gesetz vom 22.07.1998 (BGB1. I, S. 1881).

${ }^{13}$ Fünftes Buch Sozialgesetzbuch - Gesetzliche Krankenversicherung (Artikel 1 des Gesetzes vom 20.12.1988, BGB1. I, S. 2482-551, § 76 geändert durch Artikel 1 des Gesetzes vom 21.12.1992, BGB1 I, S. 2273).

${ }^{14} \mathrm{BMV}-\ddot{\mathrm{A}}, \mathrm{S} .23$.

${ }^{15}$ Vgl. BGB in der Fassung der Bekanntmachung vom 02.01.2002 (BGBl. I, S. 101).

${ }^{16}$ Vgl. JANDA (2013), S. 330.
} 
Fachgesellschaften e. V.) können die im Verkehr erforderliche Sorgfalt indizieren. Der Facharztstandard bezieht sich auf einen objektiv-typisierten Sorgfaltsmaßstab ${ }^{17}$. Dieser Versuch eines vereinheitlichten, auf Evidenz basierenden Vorgehens, also die Bezugnahme auf den „medizinischen Standard“ oder „Facharztstandard“ in Form von Empfehlungen, Richtlinien oder Leitlinien, ist jedoch nicht unproblematisch. Gemäß des Oberlandesgerichts (OLG) Naumburg wohnt den Leitlinien der Arbeitsgemeinschaft der Wissenschaftlichen Fachgesellschaften e. V. nur Informationscharakter für die Ärzte inne. Einer Bedeutung als verbindlicher Handlungsanleitung stehen Diskussionen um ihre Legitimität, unterschiedliche Qualität und Aktualität gegenüber. Diese Leitlinien können zudem aufgrund ihres abstrakten Regelungsgehalts nicht als ein Ersatz eines auf einen individuellen Fall bezogenen Sachverständigengutachtens dienen $^{18}$. Zumindest sind die Richtlinien des Gemeinsamen Bundesausschusses nach SGB V §§ 91ff. wie auch die Richtlinien anderer Gremien, besonders der Bundesärztekammer, nicht standardprägend; sie haben höchstens eine „den Standard beschreibende Wirkung“ und können so den Mindeststandard (gegenüber dem Facharztstandard mit höherem Anforderungen) anzeigen ${ }^{19}$. Gemäß eines Urteils des Bundesgerichtshofs (BGH) von 2011 sind „gesicherte medizinische Erkenntnisse“ sowohl Einsichten, die sich in Leitlinien, Richtlinien, anderweitige ausdrückliche Handlungsanweisungen niederschlagen, als auch die elementaren medizinischen Grundregeln eines Fachgebietes. Das Außerachtlassen dieser Erkenntnisse führt $\mathrm{zu}$ der Annahme eines groben Behandlungsfehlers ${ }^{20}$. Dieser sogenannte grobe Behandlungsfehler dient als Instrument zur Herstellung gleich starker Positionen im Arzthaftungsprozess und wird als ein Fehler, der aus objektiver Sicht unverständlich erscheint, da er ,einem Arzt schlechterdings nicht unterlaufen darf", verstanden ${ }^{21}$. Wie der BGH 1988 herausgestellt hat, setzt die Beurteilung der Frage eines groben Behandlungsfehlers eine Gesamtbetrachtung des Behandlungsablaufs unter Rücksichtnahme auf die gegebenen Umstände voraus ${ }^{22}$; es handelt sich dabei aber nicht um die Beurteilung des Grades subjektiver Vorwerfbarkeit (im Sinne von in der Person des handelnden Arztes liegenden Gründen), sondern um das Einnehmen einer objektiven ärztlichen

\footnotetext{
${ }^{17}$ Vgl. DEUTSCH, SPICKHOFF (2014), S. 348 (Rdnr. 557).

${ }^{18}$ Vgl. OLG NAUMBURG, Urteil vom 19.12.2001 - 1 U 46/01 (LG Halle) = Medizinrecht (MedR) 2002, S. 471. ${ }^{19}$ NEBENDAHL (2014), S. 312 (Rdnr. 1030).

${ }^{20}$ Vgl. BGH, Urteil vom 20.09.2011 - VI ZR 55/09 (OLG München) = MedR 2012, S. 450.

${ }^{21}$ HART (2012), Bearbeitung von BGH, Urteil vom 20.09.2011 - VI ZR 55/09 (OLG München) = MedR 2012, S. 451; BGH, Urteil vom 27.03.2007 - VI ZR 55/05 (OLG Karlsruhe) = Neue Juristische Wochenschrift (NJW) 2007), S. 2769; vgl. auch BGH, Urteil vom 27.04.2004 - VI ZR 34/03 (OLG Braunschweig) = NJW 2004 , S. 2012.

${ }^{22}$ Vgl. BGH, Urteil vom 08.03.1988 - VI ZR 201/87 (OLG Stuttgart) = NJW 1988, S. 1511f.
} 
Sicht $^{23}$. Das OLG Stuttgart kam zu dem Ergebnis, dass der Verstoß gegen die Behandlungsregeln der Leitlinien nicht zwingend einen groben/schweren Behandlungsfehler darstellt ${ }^{24}$. Insofern der rechtliche Standard dem medizinischen Standard entspricht, erlangen Leitlinien (haftungs-)rechtliche Verbindlichkeit lediglich aufgrund von Rezeptionsnormen (BGB § 276: siehe oben; BGB $\S \S 280$ und 823: Schadenersatz $)^{25}$. Der Sorgfaltsmaßstab hat unabhängig vom Krankenversicherungsstatus des Patienten Gültigkeit, wobei vielerorts in Zweifel gezogen wird, dass die sozialrechtlichen Rahmenbedingungen, d. h. die Leistungen der gesetzlichen Krankenversicherungen, dem medizinischen Standard entsprechen; jedenfalls kann diese Inkongruenz durch eine extensive Aufklärung des Arztes und durch die Möglichkeit von ergänzenden privatärztlichen Leistungen ausgeglichen werden ${ }^{26}$. Auch muss der Arzt erkennen können, wenn es geboten ist, aufgrund der individuellen Besonderheiten des Patienten vom medizinischen Standard abzuweichen, da unter Umständen eine Entsprechung einer Leitlinie trotz dieser patientenspezifischen Besonderheiten einen Behandlungsfehler darstellen kann; ein Abweichen vom Standard muss begründet und dokumentiert werden - zudem muss der Patient entsprechend aufgeklärt werden ${ }^{27}$. Gemäß BMV- $\ddot{A} \S 13$, Abs. 7 ist der Vertragsarzt berechtigt, die Behandlung eines volljährigen Versicherten abzulehnen, wenn dieser nicht vor der Behandlung die elektronische Gesundheitskarte vorlegt, es sei denn, dass u. a. akute Behandlungsbedürftigkeit vorliegt; im Übrigen darf der Vertragsarzt die Behandlung eines Versicherten nur in begründeten Fällen ablehnen ${ }^{28}$. Von der Subjektivierung des Sorgfaltsmaßstabs im Strafrecht kann aber nicht geschlossen werden, dass eine zu einer Körperverletzung oder zum Tod führende unterdurchschnittliche Qualifikation unter Nichtbeachtung der objektiv erforderlichen Sorgfalt straflos bleibt ${ }^{29}$. Übernimmt ein Arzt ohne Not eine Behandlung, die er bei selbstkritischer Prüfung seiner eigenen Fähigkeiten bzw. im Wissen um die ihm zur Verfügung stehenden Möglichkeiten hätte ablehnen müssen (da er den Facharztstandard nicht garantieren kann), gerät er in ein Übernahmeverschulden bzw. in die

\footnotetext{
${ }^{23}$ Vgl. BGH, Urteil vom 26.11.1991 - VI ZR 389/90 (OLG Celle) = NJW 1992, S. 755.

${ }^{24}$ Vgl. OLG STUTTGART, Urteil vom 22.02.2001 - 14 U 62/2000 (LG Ellwangen) mit Bearbeitung von KERN $=$ MedR 2002, S. 650.

${ }^{25}$ Vgl. Bearbeitung von HART (2002) des Urteils des OLG Naumburg vom 19.12.2001 - 1 U 46/01 (LG Halle) = Medizinrecht (MedR) 2002 (s. o.), S. 471.

${ }^{26}$ Vgl. JANDA (2013), S. 330 und GASSNER, STRÖMER (2012), S. 159.

${ }^{27}$ Vgl. TAUPITZ (2011), S. $392 \mathrm{f}$.

${ }^{28}$ Vgl. BMV-Ä, S. 22-23.

${ }^{29}$ Vgl. ULSENHEIMER (2015), S. 54 (Rdnr. 111).
} 
Übernahmefahrlässigkeit (vgl. H. Frister et al. $^{30}$ und - als Fallbeispiel - ein Urteil des OLG Stuttgart $\left.{ }^{31}\right)$.

\subsection{Ausgewählte Grenzen der notärztlichen Hilfeleistung}

\subsubsection{Zumutbarkeit und Erforderlichkeit}

Im Strafgesetzbuch (StGB) § 323c wird hinsichtlich der unterlassenen Hilfeleistung auf eine Ausnahme- bzw. Notfallsituation Bezug genommen, in der Hilfe nicht geleistet wird, obwohl sie erforderlich und zumutbar ist (siehe unten). Hinsichtlich objektiver Gesichtspunkte bestehen gemäß der Handreichung der Bundesärztekammer für Telefondisponenten in Notdienstzentralen und Rettungsdienstleitstellen folgende (fachspezifische) Indikationen für den Notarzteinsatz, der im Anhang zum Kommentar zum RDG Baden-Württemberg wie folgt gelistet ist: Notfallbezogene Indikationen sind 1. Schwerer Verkehrsunfall, 2. Unfall mit Beteiligung von Kindern, 3. Brände bzw. Rauchgasentwicklung mit Zeichen auf Involvierung von Personen, 4. Explosionen, thermische oder chemische Unfälle, Stromunfälle, 5. Wasserbzw. Ertrinkungsunfälle, Eiseinbruch, 6. Maschinenunfall mit Einklemmung von Personen, 7. Verschüttung von Personen, 8. Drohende Selbsttötung, 9. Sturzgeschehen aus Höhe (> $3 \mathrm{~m}$ ), 10. Schuss-, Stich-, Hiebverletzungen im Bereich des Kopfes, Halses oder Rumpfes, 11. Geiselnahme und sonstige Verbrechen mit direkter Lebensgefahr, 12. Unmittelbar beginnende oder erfolgte Geburt und 13. Vergiftungen ${ }^{32}$. Der Indikationskatalog hinsichtlich des Patientenzustandes mit abwesenden oder stark beeinträchtigen Vitalfunktionen enthält das Folgende: 1. Fehlende Reaktion auf Ansprechen bzw. Schütteln - Bewusstseinslage, 2. Schwere oder zunehmende Atemnot/Atemstillstand - Atmung; 3. Akuter Brustschmerz/deutliche oder zunehmende Kreislaufinsuffizienz/Kreislaufstillstand Herz/Kreislauf; 4. Schwere Verletzung/starke Blutung/ausgeprägte akute Schmerzen/plötzliche Lähmung (halbseitig) - sonstige Schädigungen mit Wirkung auf die Vitalfunktionen $^{33}$. Die fehlende Erforderlichkeit der notärztlichen Hilfe ist eine prinzipiell selbsterklärende und offenkundige, da aus dem medizinischen (Facharzt)-Standard hervor-

\footnotetext{
${ }^{30}$ FRISTER, LINDEMANN, PETERS (2011), S. 77 (Rdnr. 150).

${ }^{31}$ OLG STUTTGART, Urteil vom 04.01.2000 - 14 U 31/98. Anforderungen an die Überwachung Neugeborener bei Vorliegen eines erhöhten Infektionsrisikos = VersR 2001, S. 1560 ff. (Ein zugezogener Kinderarzt dürfe es nach stattgehabter Übernahme der Behandlung, sofern er für eine erforderliche Intubation eines Neugeborenen keine genügenden Kenntnisse und Erfahrungen innehabe, nicht auf einer Maskenbeatmung belassen; er hätte darauf bestehen müssen, dass ein kompetenter Krankenhausarzt die Intubation übernimmt).

${ }^{32}$ Vgl. GÜNTERT, ALBER (1999), Anhang 5a, S. 1.

${ }^{33}$ Vgl. GÜNTERT, ALBER (1999), Anhang 5a, S. 1.
} 
gehende, Grenze - zumindest aus der a posteriori-Betrachtung. In praxi, a priori wird der Notarzt bei Unsicherheit über die Notwendigkeit seiner Tätigkeit den Einsatz durchführen - um einem Vorwurf der Fahrlässigkeit (vgl. StGB $§ \S 229,222,230$ ) bzw. eines Unterlassungsdelikts/einer unterlassenen Hilfeleistung (vgl. StGB $\S \S 13,323 \mathrm{c}$ ) zu entgehen - und dann das Notwendige (z. B. Information des hausärztlichen Notdienstes) in die Wege leiten (vgl. $M$. Ufer 1999) ${ }^{34}$. Der Sicherstellungsauftrag der Kassenärztlichen Vereinigungen umschließt gemäß SGB V § 75 Abs. 1, Satz 1 und 2 nämlich auch die vertragsärztliche Versorgung zu den Zeiten ohne Sprechstunde.

Gemäß J. C. Schuhr ist die Zumutbarkeit in den konkreten, individuellen Umständen die Voraussetzung für die Hilfeleistungspflicht und als Tatbestandsmerkmal zu betrachten. Pflicht ist eine objektive Kategorie der Rechtsordnung - dem widerspricht nicht, dass die Frage der Zumutbarkeit lediglich in der konkreten Situation für die potentiell zu Hilfeleistung verpflichtet beteiligte Person, d. h. unter Abstellen auf die Besonderheiten von Situation und Person, beurteilt werden $\operatorname{kann}^{35}$. Um vom Vorliegen von Zumutbarkeit ausgehen zu können, muss daher eine objektive Gesamteinschätzung im Sinne einer Güterabwägung aller tatsächlichen Umstände des individuellen Falles bzw. der Situation, die zu einem klaren Dominieren der Interessen des Patienten führen muss, vorgenommen werden: Dies inkludiert z. B. das Ausmaß der Gefährdung bzw. die Schwere der Verletzung/Erkrankung des Hilfsbedürftigen, die Wahrscheinlichkeit zusätzlicher Schäden bzw. einer Verschlechterung seines Zustands, die fachlichen (gebotenen) Fähigkeiten des Arztes bzw. die ihm vorliegenden Hilfsmittel, die betroffenen Rechtsgüter des Verunglückten/Erkrankten, die Entfernung zum Ort des Unfalles oder andere eigene bzw. fremde schutzwürdige Belange ${ }^{36}$. Auch für den Arzt gilt die Verpflichtung, Hilfe zu leisten, welche er an dem von ihm eingenommenen Platz schuldet: „Jedermann-Hilfe“ muss er als Passant auf der Straße leisten; ärztliche Versorgung gemäß des Facharztstandards hat er in seiner Funktion als Krankenhausarzt, niedergelassener Arzt (ärztlicher Notdienst) bzw. als Notarzt zu garantieren ${ }^{37}$. Darüber hinaus wurde dem Arzt ein größeres Ausmaß an Hilfe, wenn er beispielweise bei einem Unfall zufällig vorhanden ist, als „,beschränkte Hilfspflicht“ im Rahmen von persönlichem Vermögen/zur Verfügung stehenden Hilfsmitteln zugemutet ${ }^{38}$.

\footnotetext{
${ }^{34}$ UFER (1999), S. 3f.

${ }^{35}$ Vgl. SCHUHR (2014), S. 2751f. (Rndr. 39f.).

${ }^{36}$ Vgl. ULSENHEIMER (2010), S. 1652 (Rndr. 43).

${ }^{37}$ DEUTSCH, SPICKHOFF (2014), S. 460 (Rdnr. 719).

${ }^{38}$ DEUTSCH, SPICKHOFF (2014), S. 617f. (Rdnr. 956).
} 


\subsubsection{Andere Grenzen}

Hinsichtlich der Frage nach dem einer Behandlung entgegenstehenden Willen des Patienten ist zuerst dem Verständnis der Rechtsordnung der Begriffe „Wille“ bzw. „Einwilligungsfähigkeit“" nachzugehen. Im Transplantationsgesetz $\S 8 \mathrm{a}$, Satz 1, Nr. 5 findet sich ein diesbezüglicher Ansatz: „Ist die minderjährige Person in der Lage, Wesen, Bedeutung und Tragweite der Entnahme zu erkennen und ihren Willen hiernach auszurichten, so ist auch ihre Einwilligung erforderlich“339. Hilfestellungen für die Frage nach dem rechtlichen Verständnis von Einwilligungsfähigkeit kann auch die Thematik der Geschäftsfähigkeit geben, da auch hier die Frage nach der freien Willensbildung beurteilt wird. Dies nicht zuletzt vor dem Hintergrund des Abschlusses eines Behandlungsvertrages zwischen Arzt und Patient, obwohl gemäß H. Dreßing et al. die Einschätzung der eigentlichen Geschäftsfähigkeit ausschließlich Aufgabe des Gerichts ist; der Arzt muss psychopathologische Anknüpfungstatbestände kommunizieren, aufgrund derer das Gericht feststellen kann, ob die Voraussetzungen zu einer freien Willensbildung möglicherweise nicht mehr vorliegen bzw. vorlagen ${ }^{40}$. Gemäß BGB $\S$ 104, Abs. 2 ist geschäftsunfähig, ,wer sich in einem die freie Willensbestimmung ausschlieBenden Zustand krankhafter Störung der Geistestätigkeit befindet, sofern nicht der Zustand seiner Natur nach ein vorübergehender ist ${ }^{\text {“41 }}$. Und BGB $§ 105$ besagt, dass die Willenserklärung eines Geschäftsunfähigen nichtig ist (vgl. Abs. 1) und dass auch eine „Willenserklärung, die im Zustand der Bewusstlosigkeit oder vorübergehender Störung der Geistestätigkeit abgegeben wird“" ${ }^{42}$, nichtig ist (vgl. Abs. 2). Wie Habermeyer und Saß sowie Dreßing et al. betonen, sind die Voraussetzungen zur Annahme einer freien Willensbildung dann nicht gegeben, wenn eine psychische Erkrankung oder Störung ,die Umsetzung persönlicher Wertvorstellungen verhindert. ${ }^{643}$ Die Erkrankung kann hier zum einen die kognitiven Voraussetzungen der Intentionsbildung und Prozesse der Intentionsinitiierung bzw. Intentionsrealisierung in Mitleidenschaft ziehen; zum anderem kann die Krankheit die motivationalen Voraussetzungen der Willensbildung abändern (durch Verbauung des Zugangs zu Wertvorstellungen bzw. Umformung des Wertgefüges oder der affektiven, dynamischen Grundlagen von Entscheidungsprozessen $)^{44}$.

\footnotetext{
${ }^{39}$ Transplantationsgesetz in der Fassung der Bekanntmachung vom 4. September 2007 (BGB1. I, S. 2212).

${ }^{40}$ Vgl. DRESSING, LEYGRAF, SCHNEIDER (2014), S. 1202.

${ }^{41}$ BGB in der Fassung der Bekanntmachung vom 02.01.2002 (BGB1. I, S. 83f.).

${ }^{42}$ BGB in der Fassung der Bekanntmachung vom 02.01.2002 (BGB1. I, S. 84).

${ }^{43}$ HABERMEYER, SASS (2002), S. 8 und DRESSING, LEYGRAF, SCHNEIDER (2014), S. 1202.

${ }^{44}$ Vgl. HABERMEYER, SASS (2002), S. 8 und DRESSING, LEYGRAF, SCHNEIDER (2014), S. 1202.
} 
Wie A. Laufs und B.-R. Kern unterstrichen haben, kann ein Behandlungsfehler nicht nur durch ein „Zuviel“ (d. h. ein Tun), sondern auch durch ein „Zuwenig“ (d. h. ein Unterlassen) zustande kommen; somit besteht hinsichtlich der Verantwortlichkeit des Arztes kein Unterschied dahingehend, ob das Gewicht des Handelns des Arztes in der Durchführung einer sachwidrigen oder in dem Unterlassen einer der Sache nach geforderten Maßnahme liegt ${ }^{45}$. Gemäß BÄO § 1 , Abs. 1 dient der Arzt „,der Gesundheit des einzelnen Menschen und des gesamten Volkes“46. Ähnlich sagt die MBO $§ 1$, Abs. 1, dass Ärztinnen und Ärzte der Gesundheit des einzelnen Menschen und der Bevölkerung“ dienen; und Abs. 2 besagt, dass es Aufgabe der Ärztinnen und Ärzte ist, „,das Leben zu erhalten, die Gesundheit zu schützen und wiederherzustellen, Leiden zu lindern, Sterbenden Beistand zu leisten und an der Erhaltung der natürlichen Lebensgrundlagen im Hinblick auf ihre Bedeutung für die Gesundheit der Menschen mitzuwirken. ${ }^{\text {"47 }}$ Wie die MBO $\S 11$, Abs. 1 besagt, verpflichten sich die Ärzte gegenüber den Patienten jedenfalls ,zur gewissenhaften Versorgung mit geeigneten Untersuchungs- und Behandlungsmethoden. ${ }^{\text {“48 }}$ Der Arzt schuldet dem Patienten das Bemühen um die Heilung gemäß des Facharztstandards, nicht aber den Erfolg, d. h. die Heilung als solche. Schicksalhafte krankhafte Entwicklungen bzw. suboptimale Resultate trotz Einhalten des jeweiligen medizinischen Standards können daher dem Arzt nicht als Behandlungsfehler zugerechnet werden ${ }^{49}$.

In ähnlicher Weise gibt es biologische oder räumliche Grenzen (Grenzen der Natur der Sache). So spricht z. B. der RDP, Kapitel III, Abschnitt 1 von der Hilfsfrist (d. h. die Zeitdauer vom Eintreffen der Notfallmeldung in der Integrierten Leitstelle bis zum Ankommen der Hilfe am Ort der Notfallsituation an Straßen) als einer „Planungsgröße, auf deren Grundlage die rettungsdienstlichen Strukturen in den Rettungsdienstbereichen festzulegen sind. ${ }^{\text {"50 }}$ Diese soll sich aus Gründen der Notfallmedizin - wenn möglich - auf nicht mehr als 10 Minuten, im äußersten Fall aber auf 15 Minuten belaufen (vgl. die gesetzliche Grundlage in RDG $§ 3$, Abs. 2). Die Vorbedingungen hierfür (d. h. das dem Bedarf entsprechende Errichten von Notfallrettungssystemen, personell ausgestattet gemäß RDG § 9) hat planerisch der Bereichsausschuss

\footnotetext{
${ }^{45}$ LAUFS, KERN (2010), S. 1204 (Rdnr. 30).

${ }^{46} \mathrm{~B} \ddot{\mathrm{A} O}$ vom 16.04.1987 (BGB1. I, S. 1219).

${ }^{47}$ MBO, S. 2.

${ }^{48} \mathrm{MBO}$, S.4.

${ }^{49}$ Vgl. JANDA (2013), S. 322, OLG Zweibrücken, Urteil vom 10.02.1998 - 5 U 65/96 = NJWE-VHR 1998, 237f. und LAUFS, KERN (2010), S. 1200 (Rdnr. 7).

${ }^{50}$ RDP, S. 158.
} 
zu herzustellen (vgl. RDP Kapitel VIII, Abschnitt 1) ${ }^{51}$. Laut RDP Kapitel III, Abschnitt 2 gibt es aufgrund besonderer Umstände keine Hilfsfristgebundenheit für die Luftrettung sowie den Berg- und Wasser-Rettungsdienst ${ }^{52}$.

Ausgehend von einer möglichen Konfliktsituation, die darin besteht, dass der Notarzt in die Situation gerät, welchem von zwei gleich schwer Verletzten (aus einer ex ante-Sicht gesehen) zu helfen, besagt die rechtfertigende/entschuldigende Pflichtenkollisionen den Umstand, dass sich der Notarzt nur einem von diesen zwei Verletzen gemäß seines medizinischen Standards straffrei widmet ${ }^{53}$. Eine Indikation zum Einsatz des Leitenden Notarztes (laut RDP, siehe auch oben) besteht bei einem Ungleichgewicht zwischen dem notfallmedizinischen Versorgungsbedarf und dem aktuellen Vermögen des Rettungsdienstes; damit ist eine individualmedizinischen Betreuung gemäß des gebräuchlichen rettungsdienstlichen Kriteriums bzw. Standards zumindest vorübergehend nicht mehr möglich ${ }^{54}$.

\subsection{Lösungsansätze der konkreten Fragestellung}

Der geschichtliche Werdegang der allgemeinen, d. h. für Jedermann geltenden, Nothilfevorschrift ist von $M$. Barthel detailliert beleuchtet worden ${ }^{55}$. Der Rechtszustand in der Bundesrepublik Deutschland von 1953 wird durch § 330c StGB wie folgt wiedergeben:

„Wer bei Unglücksfällen oder gemeiner Gefahr oder Not nicht Hilfe leistet, obwohl dies erforderlich und ihm den Umständen nach zuzumuten, insbesondere ohne erhebliche eigene Gefahr und ohne Verletzung anderer wichtiger Pflichten möglich ist, wird ... bestraft. ${ }^{\text {‘56 }}$

Damit wurde der vor 1953 bestehende Bezug zur Hilfeleistung nach polizeilicher Aufforderung entfernt. Die Zumutbarkeitsklausel wurde in den folgenden Änderungsvorschlägen der Entwürfe im Rahmen von Reformbemühungen im Wesentlichen nicht berührt. 1980 wurde durch Gesetz lediglich die Stellung der Vorschrift im StGB verändert (§ 330c wurde zu § $323 \mathrm{c})^{57}$. Dies ist einschlägig für die im vorliegenden Fall bestehende Situation, und zwar insofern, dass nämlich der ,zuständige Einsatzführer der vor Ort tätigen Polizeikräfte das Rettungsteam ... in das Objekt entsenden möchte“. Dieses Gesetz enthält also keine

\footnotetext{
${ }^{51}$ Vgl. RDP, S. 174.

${ }^{52} \mathrm{Vgl}$. RDP, S. 158.

${ }^{53}$ Vgl. UFER (1999), S. 6.

${ }^{54}$ Vgl. RDP, S. 162.

${ }^{55}$ BARTHEL (2004), S. 47ff.

${ }^{56}$ Drittes Strafrechtänderungsgesetz vom 04.08.1953 (BGB1. I, S. 743).

${ }^{57}$ Achtzehntes Strafrechtänderungsgesetz - Gesetz zur Bekämpfung der Umweltkriminalität - vom 28.03.1980 (BGB1. I, S. 374).
} 
Weisungsbefugnis der Polizei gegenüber dem Rettungsteam, betrachtet aus der Perspektive einer allgemeinen und damit auch den Arzt und das Personal der Rettung betreffenden Hilfeleistungspflicht.

Gemäß dem Landeskatastrophenschutzgesetz (LKatSG) § 20, Abs. 1 leitet der technische Leiter des Einsatzes nach den Weisungen der Katastrophenschutzbehörde die Katastrophenbekämpfung am Ort des Einsatzes, wobei ihm alle dort vorhandenen Einsatzkräfte mit ihrem Führungspersonal für die Zeit des Einsatzes unterstellt sind; ob hierunter auch die Einsatzkräfte des Rettungsdienstes mit dem Leitenden Notarzt subsumiert werden, wird nicht explizit gesagt - die Formulierung ,alle dort eingesetzten Einsatzkräfte“658 legt aber Selbiges nahe. Auch wird das Leiten nicht mehr weiter differenziert (z. B. in Fragen nach der Organisation des Einsatzes oder eher auch Gesichtspunkte der medizinischen Versorgung im engeren Sinne). LKatSG $\S 20$, Abs. 3 besagt, dass zum technischen Leiter entweder ein Führer einer Einheit bzw. Einrichtung des Katastrophenschutzdienstes oder ein feuerwehrtechnischer Beamter (gemäß $§ 23$ des Feuerwehrgesetzes ${ }^{59}$ ) bestellt werden soll; in speziellen Fällen kann ein Beamter der Polizei, ein Bediensteter einer Behörde oder ein Angehöriger eines Betriebes (falls dessen Fachkenntnisse bei der Bekämpfung der Katastrophe besondere Wichtigkeit innehaben) zu dieser Funktion bestellt werden. Demgemäß können auch Polizeikräfte über diesen Weg der Bestellung als technische Leiter maßgebliche Entscheidungsbefugnis für den gesamten Einsatz erhalten. Bei einem Großschadensfall mit einem zur gleichen Zeit stattfindenden Einsatz nach dem Feuerwehrgesetz formt der Feuerwehrkommandant gemäß des RDP einen örtlichen Einsatzstab, in den der Leitende Notarzt und der Organisatorische Leiter Rettungsdienst eingegliedert sind, wobei dem Leitenden Notarzt die Führung der medizinischen Notfallversorgung obliegt ${ }^{60}$. Letzterer ist Mitglied im Stab des Technischen Einsatzleiters bei Vorliegen einer Katastrophe (vgl. LKatSG $\S 20$, Abs. 2). Laut RDP hat der Leitende Notarzt Weisungsbefugnis nicht nur gegenüber den involvierten Ärzten (siehe oben) und dem Personal des Rettungsdienstes, sondern - bei Delegation durch den Technischen Einsatzleiter auch gegenüber dem Sanitätspersonal des Katastrophenschutzes ${ }^{61}$. Das gegenwärtige Fallbeispiel mit einer unübersichtlichen polizeilichen Situation bei einer Amoklage schließt einen Katastrophenfall im Sinne des LKatSG, definiert als ein „Geschehen, das Leben oder Gesundheit zahlreicher Menschen oder Tiere, die Umwelt, erhebliche Sachwerte oder die

\footnotetext{
${ }^{58}$ Gesetz über den Katastrophenschutz in der Fassung vom 22.11.1999 (GB1. 1999, S. 631).

${ }^{59} \mathrm{Vgl}$. Feuerwehrgesetz (FWG) in der Fassung vom 02.03.2010 (GB1. 2010, S. 341).

${ }^{60}$ Vgl. RDP, S. 162 und RDG $§ 10$, Abs. 2.

${ }^{61}$ Vgl. RDP, S. 162.
} 
lebensnotwendige Versorgung der Bevölkerung in so ungewöhnlichem Maße gefährdet oder schädigt, dass es geboten erscheint, ein zu seiner Abwehr und Bekämpfung erforderliches Zusammenwirken von Behörden, Stellen und Organisationen unter die einheitliche Leitung

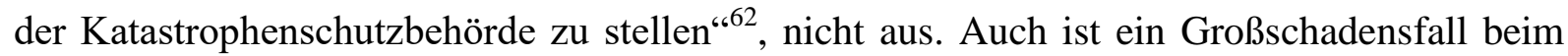
vorliegenden Fallbeispiel möglich (Plural: „lebensbedrohlich Verletzen“). Das in dieser Arbeit bereits Ausgeführte (siehe oben) würde in diesen Fällen dann für die Beziehungen der leitenden Positionen der Rettung, Feuerwehr, Polizei und des Katastrophenschutzes gelten. Jedenfalls haben sich, wie der RDP ausführt, die Einsatzleiter von Feuerwehr und Rettung gegenseitig ,zu unterstützen, eng zusammen zu arbeiten [sic] und ihre Einsatzmaßnahmen abzustimmen.“63 Aus der berufsrechtlichen Perspektive heraus gesehen gebietet MBO $\S 2$, Abs. 4, dass ärztliches Personal hinsichtlich seiner ärztlichen Entscheidungen keine Anweisungen von Nichtärzten annehmen darf.

Die Anlage 24 BMV-Ä $\S 2$ besagt, dass der Arzt Leistungen, die er aufgrund der erforderlichen besonderen Fachkenntnisse nur persönlich erbringen kann, auch nicht delegieren darf. Dazu gehören u. a. Indikationsstellung, Diagnosestellung, Aufklärung und Beratung des Patienten und Entscheidungen über die Therapie. Obwohl die Thematik dieser Vereinbarung die Anforderungen an die Delegation ärztlicher Leistungen an nichtärztliche Mitarbeiter - und nicht die eigentliche Weisungsbefugnis - ist, bringt es doch den Umstand zum Ausdruck, dass höchstpersönliche, durch den Arzt zu erbringende Leistungen, wie das Stellen der Indikation eines ggf. weiteren (medizinischen) Einsatzes, eben eine höchstpersönliche Entscheidung des Arztes gemäß des medizinischen Standards ist, und nicht der sinnvolle Inhalt einer Weisung von nichtärztlichem Personal an Ärzte sein kann - schon aufgrund der mangelnden Fachkenntnis des nichtärztlichen Personals nicht ${ }^{64}$.

Im vorliegenden Fall liegen lebensbedrohliche Verletzte (Schussverletzung) im Sinne einer schweren Verletzung mit Bedrohung der Vitalfunktionen (vgl. Handreichung der Bundesärztekammer) $\operatorname{vor}^{65}$. Dieses Fallbeispiel fügt sich auch in die von der WBO geforderten Inhalte (siehe oben): z. B. Diagnose/Therapie akuter Störungen der Vitalfunktionen, Notfallmedikation, sachgerechte Lagerung und Herstellung der Transportfähigkeit von Not-

\footnotetext{
${ }^{62}$ Gesetz über den Katastrophenschutz in der Fassung vom 22.11.1999, (GB1. 1999, S. 625 ff.), geändert durch Artikel 5 des Gesetzes zur Änderung des Gesetzes zur Ausführung des Tierseuchengesetzes und anderer Gesetze vom 11.03.2004 (GB1., S. 114).

${ }^{63}$ RDP, S. 162.

${ }^{64}$ ANLAGE 24 BMV-Ä, S. 2.

${ }^{65}$ GÜNTERT, ALBER (1999), Anhang 5 a, S. 1.
} 
fallpatienten sowie evtl. auch Besonderheiten beim Massenanfall Verletzter und Erkrankter einschließlich Sichtung ${ }^{66}$. Schussverletzungen sind auch in der S3-Leitlinie Polytrauma/Schwerverletzten-Behandlung, allerdings im Rahmen von sogenannten Schockraumaktivierungskriterien, enthalten ${ }^{67}$. Es lassen sich also objektivierbare Argumente finden, die vom medizinischen bzw. Facharztstandard gesehen den Einsatz des Notarztes erforderlich machen würden, und bei denen insofern der Notarzt auch zum Einsatz verpflichtet wäre. Das erwähnte „Höchstpersönliche“ der Leistungserbringung, wie z. B. das Anordnen des diagnostischen und therapeutischen Prozederes, die Selbständigkeit, Eigenverantwortlichkeit und fachliche Unabhängigkeit des Arztes - die in seiner Stellung als Freiberufler zum Ausdruck kommt kennzeichnen aber, dass es unter Berücksichtigung der konkreten, individuellen Gesamtsituation im Eigentlichen die Entscheidung des Arztes ist, ob er den Einsatz persönlich, unter eventueller eigener Gefährdung vornehmen kann. Die Gefährdung bzw. Verletzung des Notarztes als handelndes Subjekt des Einsatzes würde auch den Einsatz als solchen bedrohen, und objektivierbare, typisierte Sorgfaltsforderungen des Arztes gegenüber dem Patientin würden von vornherein stark beeinträchtigt oder verunmöglich werden. Die MBO $\S$ 2, Abs. 1 besagt, dass Ärzte ihren Beruf nach ihrem „Gewissen“ ausüben und dass sie keine Grundsätze anerkennen und keine Vorschriften oder Anweisungen beachten dürften, „die mit ihren Aufgaben nicht vereinbar sind oder deren Befolgung sie nicht verantworten können. “68 Die $§$ 7-12 der MBO haben, als nähere Ausformung, die Pflichten der Ärzte gegenüber ihren Patienten zum Inhalt.

\section{$3 \quad$ Fazit}

Bei der prinzipiellen Beurteilung der im Fallbeispiel aufgeworfenen Fragen, ob nämlich der Einsatz des Notarztes erforderlich und v. a. zumutbar ist und ob die Gefährdungssituationen „nur“ durch den Notarzt empfunden werden oder real einer allgemeinen Wahrnehmung entsprechen (die aber letztlich auch ein Empfinden, wenn auch ein Generelleres sein kann), ist objektiv-typisierend auf den Facharztstandard bzw. den Indikationskatalog für den Notarzteinsatz in Kombination mit arzt-/berufsrechtlichen Gesichtspunkten im engeren Sinne (z. B. Freiberuflichkeit) abzustellen. Es wird sich jedenfalls immer um eine Einzelfallbetrachtung

\footnotetext{
${ }^{66} \mathrm{Vgl}$. WBO, S. 103.

${ }^{67} \mathrm{Vgl}$. DEUTSCHE GESELLSCHAFT FÜR UNFALLCHIRURGIE (2011), S. 147.

${ }^{68} \mathrm{MBO}$, S. 2.
} 
und um eine individuelle, fallbezogene Entscheidung handeln. Bei seltenen, sehr unwahrscheinlichen Situationen mit relativer Ermangelung von objektiv-typisierbaren Gesichtspunkten werden die Anforderungen, aber auch die Chancen, an die „höchstpersönliche“ Berufsausübung, an die Persönlichkeit des Arztes (v. a. in der Entscheidungsfindung) umso größer werden. 


\section{Literaturverzeichnis}

\section{Schrifttum:}

Barthel M.: Die (Un-)Zumutbarkeit des erfolgsabwenden Tuns. Begriff und systematische Einordnung. Dissertation zur Erlangung des Doktorgrades der Rechtswissenschaftlichen Fakultät der Friedrich-Schiller-Universität Jena. TENEA Verlag für Medien, Juristische Reihe TENEA, Bd. 66, 2004.

Deutsch E., Spickhoff A.: Medizinrecht. Arztrecht, Arzneimittelrecht, Medizinprodukterecht und Transfusionsrecht. Springer-Verlag Berlin Heidelberg, 7. Auflage, 2014.

Deutsche Gesellschaft für Unfallchirurgie (federführend, Hrsg.). S3 - Leitlinie Polytrauma/Schwerverletzten-Behandlung, AWMF-Register Nr. 012/019: AWMF online, 2011, abrufbar unter:

<http://www.awmf.org/uploads/tx_szleitlinien/012-0191_S3_Polytrauma_SchwerverletztenBehandlung_2015-01.pdf> (zugegriffen am 23.05.2016).

Dreßing H., Leygraf J., Schneider F.: Begutachtung der Geschäfts- und Testierfähigkeit. Eine komplexe Aufgabe für den Arzt. Dtsch Arztebl, 2014, Ausgabe A, 1202-4.

Frister H., Lindemann M., Peters A.: Praxis des Medizinrechts. Arztstrafrecht. Verlag C.H. Beck, München, 2011.

Gaßner M., Strömer J. M.: Die Arzthaftung bei der Behandlung gesetzlich krankenversicherter Patienten. MedR 2012, S. 159-69.

Bundesärztekammer: Indikationskatalog für den Notarzteinsatz. Handreichung der Bundesärztekammer für Telefondisponenten in Notdienstzentralen und Rettungsdienstleitstellen vom 23.11.2001, Baden-Württemberg 2003 / 2. Nachlieferung Dezember 2003, Anhang 5a zu: Güntert L., Alber W. (begründet), Lottermann A. (fortgeführt): Rettungsdienstgesetz BadenWürttemberg. Kommentar. Kommunal- und Schul-verlag, Wiesbaden 1999, S. 1-2. 
Habermeyer E., Saß H.: Ein am Willensbegriff ausgerichteter, symptomorientierter Ansatz zur Prüfung der Geschäftsfähigkeit. Fortschr Neurol Psychiat 2002, S. 5-10.

Heberer J.: Das ärztliche Berufs- und Standesrecht. ecomed verlagsgesellschaft, Landsberg/Lech, 2. Auflage, 2001.

Innenministerium Baden-Württemberg: Rettungsdienstplan 2014 Baden-Württemberg. 18.02.2014 - Az.: 4-5461.2-5, GABl. vom 30.04.2014, S. 156-178.

Janda C.: Medizinrecht. UVK Verlagsgesellschaft, Konstanz mit UVK/Lucius, München, 2. Auflage, 2013.

Kassenärztliche Bundesvereinigung (K. d. ö. R., Berlin), GKV-Spitzenverband (Spitzenverband Bund der Krankenkassen, K. d. ö. R., Berlin). Bundesmantelvertrag - Ärzte gemäß § 82 Abs.1 SGB V vom 01.01.2015; Anlage 24, Vereinbarung über die Delegation ärztlicher Leistungen an nichtärztliches Personal in der ambulanten vertragsärztlichen Versorgung gemäß $§ 28$ Abs. 1 S. 3 SGB V vom 01.10.2013, Stand: 01.01.2015.

Nebendahl M. 10. Kapitel Arzthaftungsrecht. In: Igl G., Welti F.: Gesundheitsrecht. Eine systematische Einführung, Verlag Franz Vahlen, München, 2. Auflage, 2014, S. 247-402.

Laufs A, Kern B.-R.: 17. Kapitel. Die vertragliche Haftplicht des Arztes und des Krankenhausträgers. In: Laufs A, Kern B.-R.: Handbuch des Arztrechts. Verlag C.H. Beck, München, 4. Auflage, 2010, S. 1179-241.

Schuhr J. C.: 600. Strafgesetzbuch (StGB). Achtundzwanzigster Abschnitt. Gemeingefährliche Straftaten. § 323c Unterlassene Hilfeleistung. In: Spickhoff A. (Hrsg.): Medizinrecht. Beck'sche Kurz-Kommentare, Band 64, Verlag C. H. Beck, München, 2. Auflage, 2014, S. $2743-57$.

Taupitz J. Medizinische Informationstechnologie, leitliniengerechte Medizin und Haftung des Arztes. AcP, 2011, S. 352-94. 
Ufer M. R.: Grenzen der präklinischen Notfallmedizin aus juristischer Sicht. Anästhesiol Intensivmed Notfallmed Schmerzther 1999, S. 3-9.

Ulsenheimer K.: Kap. 1, Teil 1 Fahrlässige Tötung (§ 222 StGB) und fahrlässige Körperverletzung (§ 229 StGB). In: Ulsenheimer K.: Arztstrafrecht in der Praxis. Praxis der Strafverteidigung, Band 7. C. F. Müller, einer Marke der Verlagsgruppe Hüthig Jehle Rehm, Heidelberg, München, Landsberg, Frechen, Hamburg, 5. Auflage, 2015, S. 19-376.

Ulsenheimer K.: 23. Kapitel. Der Arzt im Strafrecht. In: Laufs A., Kern B.-R.: Handbuch des Arztrechts. Verlag C. H. Beck, München, 4. Auflage, 2010, S. 1569-878.

\section{Entscheidungen:}

BGH: Urteil vom 08.03.1988 - VI ZR 201/87 (OLG Stuttgart) = Voraussetzungen für Annahme eines groben Behandlungsfehlers, NJW 1988, S. 1511-3.

BGH: Urteil vom 26.11.1991 - VI ZR 389/90 (OLG Celle) = Beweiserleichterung bei grobem Behandlungsfehler keine Sanktion für besonders schweres Arztverschulden, NJW 1992, S. 754-6.

BGH: Urteil vom 27.04.2004 - VI ZR 34/03 (OLG Braunschweig) = Umkehr der objektiven Beweislast bei grobem Behandlungsfehler, NJW 2004, S. 2011-3.

BGH: Urteil vom 27.03.2007 - VI ZR 55/05 (OLG Karlsruhe) = Arzthaftung bei Behandlung mit in der Zulassungsphase befindlichem Medikament, NJW 2007, S. 2767-71.

BGH: Urteil vom 20.09.2011 - VI ZR 55/09 (OLG München) = Zur Bedeutung von Leitlinien, Richtlinien, anderweitigen ausdrücklichen Handlungsanweisungen und elementaren medizinischen Grundregeln bei der Feststellung eines groben Behandlungsfehlers. Bearbeitet von Hart, D. MedR 2012, S. 450-4. 
OLG Naumburg: Urteil vom 19.12.2001 - 1 U 46/01 (LG Halle) = Zur Bedeutung der Leitlinien von Fachgesellschaften als ärztliche Handlungsanleitung; Pflichtwidrigkeit einer Indikation. Bearbeitet von Hart, D. MedR 2002, S. 471-2.

OLG Stuttgart: Urteil vom 22.02.2001 - 14 U 62/2000 (LG Ellwangen) = Leitlinien und Standard. Bearbeitet von Kern, B.-R. MedR 2002, S. 650-4.

OLG Stuttgart: Urteil vom 04.01.2000 - 14 U 31/98 = Anforderungen an die Überwachung Neugeborener bei Vorliegen eines erhöhten Infektionsrisikos. VersR 2001, S. 1560-4.

OLG Zweibrücken, Urteil vom 10.02.1998 - 5 U 65/96 = HNO-Facharztkonsultation bei Morbus Ménière nach Gallenoperation. NJWE-VHR 1998, S. 237-8. 